\title{
STUDY OF POWER FILTER TOPOLOGIES AND CONTROL MECHANISM
}

\author{
Divya Prabha \\ MTech Scholar \\ Department of EX, OCT, Bhopal
}

\begin{abstract}
Power system comprises of three natural / physical characteristics namely voltage, current and frequency. Deviation in these physical characteristics are termed as power quality issues in power system. Presence of nonlinear current or nonlinear/unbalanced voltages and frequencies are termed as power quality issue. These (current, voltage and frequencies) deviations result in failure/damage of equipment in power system. Power electronic converters associated with their nonlinear switching characteristics and high frequency operation are the major cause for power quality issues. In order to reduce harmonics and improve power quality, Hybrid Active Power Filter (HAPF) or shunt HAPF can be employed. The power improvement can be done using various algorithm like RLS algorithm, DC link voltage controller, fuzzy logic based hybrid filter.
\end{abstract}

Keywords: RLS algorithm, Hybrid Active Power Filter, PWM

\section{INTRODUCTION}

The study of the quality of energy (PQ) has become an important issue for both companies and consumers as they increasingly increase non-linear loads and the diffusion of renewable energy in the transmission and distribution networks. The quality of the energy is mainly influenced by the current harmonics, which are fed to the distribution network by means of non-linear loads. These harmonics would also generate harmonics in the system voltage, which in turn would prevent the structures from functioning in the distribution network. These PQ disturbances are propagated to consumer devices connected to the common coupling point (PCC). This propagation of interferences can cause overheating of motors, transformers, cables, compensation capacitors and malfunction of protection devices [4].

Some attempts have been made to solve this type of problem with the quality of the power of the IMF facilities. It is possible to analyze the interharmonic

\author{
Lalit Jain \\ Professor \\ Department of EX, OCT, Bhopal
}

phenomena generated by seedless FMI and the use of active power hybrid filters (HAPF) is recommended to attenuate the interharmonic components [5].

The passive filter has been designed to compensate for the seventh harmonic and a fixed amount of reactive power required by the load. The other harmonic components and the remaining reactive power must be compensated by the APF [6].

SPQC. One type of power filter is characterized by perfect harmonic isolation, excellent filter performance, reduced passive power filter branches, simplified passive filter design, small nominal capacity and two types of typical harmonic sources [8].

\subsection{Converter-Based Classification}

During the development of AF, two types of converters are used. The inverted pulse width modulation (PWM) bridge structure currently provided acts as a nonsinusoidal current source to meet the harmonic current requirement of the non-linear load. A diode is used in series with the self-switching device (IGBT) to block the reverse voltage [4].

However, GTO-based configurations do not require the serial diode, but have a limited switching frequency. They are considered sufficiently reliable, but have higher losses and require higher values of $\mathrm{AC}$ power capacitors in parallel. Also, they cannot be used in multi-level or multi-step mode to improve performance at higher levels. 


\section{IJOSTHE}

\section{ISSN: 2349-0772 | Volume 5 Issue 4 August 2018|www.ijosthe.com}

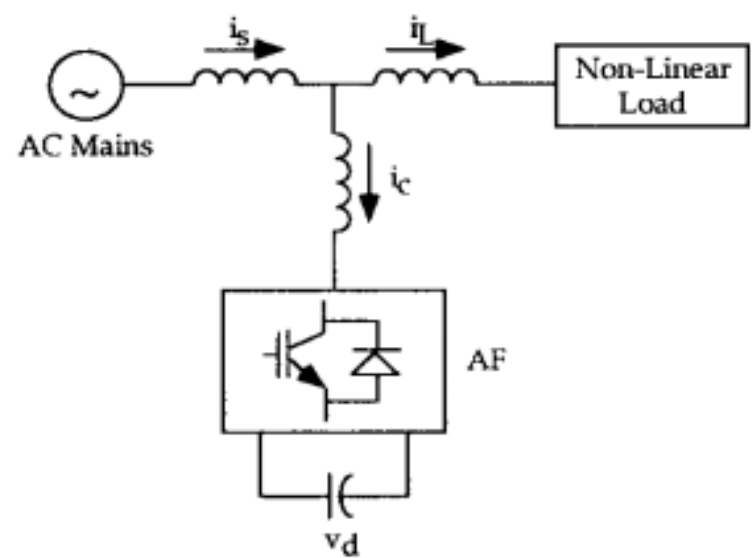

Fig. 1: Voltage-fed-type AF [4]

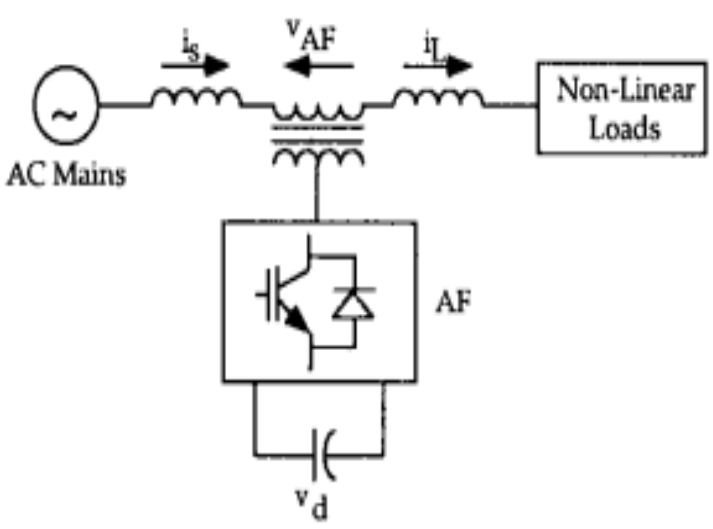

Fig. 2: Series-type AF [4]

It has become more dominant since it is simpler, more economical and extensible in multilevel and multistep versions. It is more popular in UPS-based applications, because if a network is present with the same inverter, it is possible to eliminate the harmonics of critical nonlinear loads.

\section{B. Topology-Based Classification}

AFs can be classified according to the topology used as a bypass filter or series and the unit's performance conditioners use a combination of both. Series and active combinations of passive bypass filter are known as hybrid filters. Figure 2 is an example of an active tap filter that is widely used to eliminate current harmonics, reactive power compensation (also called STATCON) and balanced asymmetric currents. It is used mainly at the end of the load because current harmonics are injected by non-linear loads.

Injects the equal compensation currents that are opposite in phase to cancel the harmonics and / or the reactive components of the non-linear load current at the connection point. It can also be used as a static var generator (STATCON) in the electrical network to stabilize and improve the voltage profile. Figure 1.2 shows the basic block of an independent active serial filter. It is placed in series with the gate before the load, using an adaptation transformer to eliminate the voltage harmonics [8] and balance and regulate the voltage of the load or the line terminal. It has been used to reduce the negative sequence voltage and to adjust the voltage in three-phase systems.

It can be installed with electrical equipment to compensate for voltage harmonics and to dampen the harmonic propagation caused by resonance with line impedances and passive bypass compensators.

Figure 3 shows a uniform power quality conditioner (also known as universal AF), which is a combination of active bypass filters and active series. It is considered an ideal AF that eliminates the harmonics of voltage and current and provides clean energy to critical and harmonic loads such as computers, medical equipment and more.

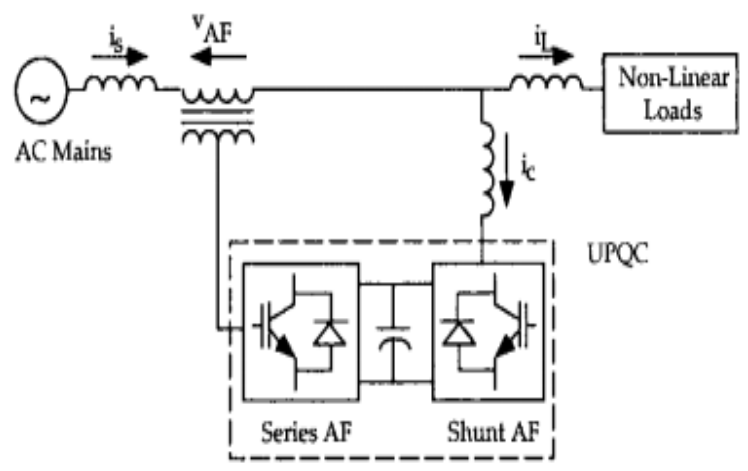

Fig. 3: Unified power quality conditioner as universal AF

You can equalize and adjust the terminal voltage and eliminate negative sequence currents. The main disadvantages are the high complexity of the costs and controls due to the large number of solid state devices involved. 


\section{IJOSTHE}

\section{ISSN: 2349-0772 | Volume 5 Issue 4 August 2018|www.ijosthe.com}

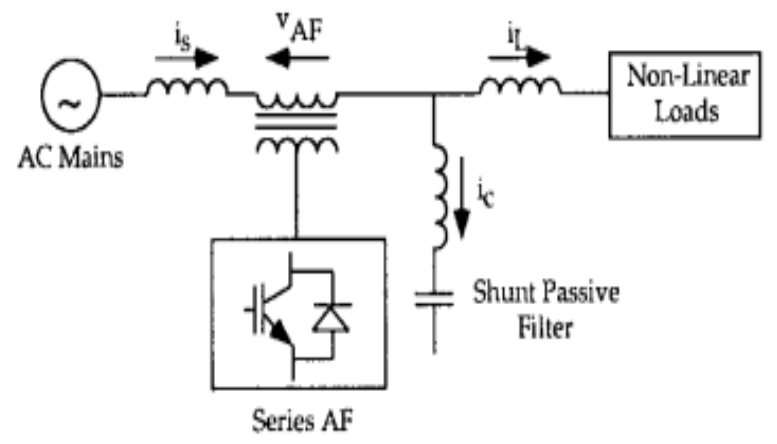

Fig. 4: Hybrid filter as a combination of active series and passive shunt filters

Figure 4 shows the hybrid filter, which is a combination of an active-series filter and a passive-bypass filter. It is very popular because the semiconductor devices used in the active part of the series can be of reduced size and cost (approximately 5\% of the size of the load) and a large part of the passive LC hybrid passive filter is used to lower the harmonics.

\section{LITERATURE SURVEY}

[1] Soumya Ranjan Das et al. In this work, a comparative study of the hybrid HAPF model based on current control techniques, such as the $\mathrm{d}-\mathrm{q}$ and $\mathrm{p}-\mathrm{q}$ theory under different operating conditions, is carried out. Once again, these results are compared with a new estimation technique, commonly called harmonic estimation of recursive least squares (RLS) in this document. The amplitude and phase angle of the fundamental and harmonic currents are estimated using the proposed RLS technique. At the point of common coupling (PCC), the harmonic composite signal is estimated with and without HAPF. Finally, the observation that the load current and the supply current are significantly improved is analyzed with a reduction of the total harmonic distortion (THD) in different operating scenarios.

[2] T.S.Haugan et al. study the use of conservative power theory for active derivation compensation and provide experimental validation of reactive compensation and harmonic filtering. The objective is to study how the theory of conservative power functions as a control algorithm for a derivation compensator. In addition, some of the challenges associated with the practical implementation of active filters are discussed.

[3] O.P.Mahela and A.G.Shaik This article aims to provide a global view of the state of the topological aspects of the techniques used to improve the quality of energy in distribution networks for researchers, designers and engineers working in this field. This review allows selecting a PQ improvement technique adapted to a specific application in terms of technical and economic aspects. We have analyzed, classified and listed severely more than 300 research works on the PQ improvement technique of the prior art for a quick reference.

[4] Y. Jiang, J.Chang and S.Tian In this work, a new mixed algorithm of genetic theory and particle swarm optimization (GA-PSO) has been proposed to solve the design problem. Optimal hybrid active power filter (HAPF). in the design of its parameters and the optimization of investments, taking into account the best convergence of the genetic theory and the rapid convergence of the optimization of the particle swarm. It requires initial investment, reactive power compensation capacity and harmonic distortion as objectives, and the theory of the penalty function has been used to convert multiple-goal design problems into design problems. single objective Finally, a simulation of HAPF was analyzed in the context of PSCAD / EMTDC. The results show that the optimal design method proposed by HAPF can reduce costs, improve the price/performance ratio and improve filter performance.

\section{METHODOLOGY}

Because of its simplicity and best performance, RLS algorithms are widely used in many applications. The generation of a compensation current using the RLS algorithm is used in HAPF to obtain the DC link active current (Adcx) parameter, keep the intermediate circuit voltage at the constant value of each phase and the waveform in the wave form. The HAPF can reach the value of the instantaneous active current to compensate for the loss of power and the difference in active power between the load and the power system. Figure 2 shows the operation of the RLS algorithm, in which the voltage of the intermediate reference circuit Vref and Vdc (i) the intermediate circuit voltage has a current sample. There are no harmonics in the voltage of the source, so the instantaneous voltage is given as follows: [1]:

$$
V_{x}(t)=V_{a x}(t)
$$

For each phase, the reference active current is given as 


\section{ISSN: 2349-0772 | Volume 5 Issue 4 August 2018|www.ijosthe.com}

$$
i_{a x}(t)=A_{x} V_{x}(t)
$$

The instantaneous reference compensating current specified as

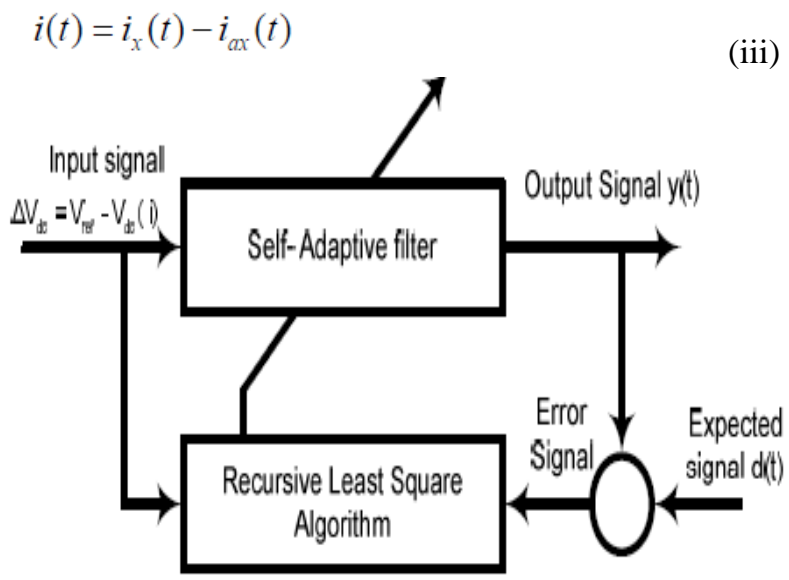

Fig. 5: Flow chart of RLS filter

Intermediate circuit voltage regulator The HPF operates at a fixed intermediate circuit voltage level. Since the switching loss is directly proportional to the DC link voltage, the system achieves a greater switching loss when a higher DC voltage is used and vice versa.

For a fixed intermediate circuit voltage level for HPF using three-phase VSI [2], Vinv $=0.5 \mathrm{Vdc} / 2$ and the modulation index $\mathrm{m}$ is assumed to be $\mathrm{m} \geq 1, \mathrm{RVdc}$ represents the ratio of the intermediate circuit voltage a Load the reference voltage in neutral n, as in Eq. iv.

$R_{V_{d c}}=\frac{ \pm V_{i n v}}{V_{x}}=\frac{ \pm 0.5 V_{d c} / \sqrt{2}}{V_{x}}=\frac{ \pm V_{d c}}{2 \sqrt{2} V_{x}}$

where $V_{\text {inv }}$ is the inverter fundamental RMS voltage, and $V_{x}$ is phase rms supply voltage. Similarly, for HAPF using nine-switch inverter [9] the dclink voltage is given by Eqn 3 .

$$
V_{d c}=2 \sqrt{2} V_{x}
$$

For $\mathrm{APF}, \mathrm{R}_{\mathrm{Vdc}}>1$ and for $\mathrm{HAPF}, \mathrm{R}_{\mathrm{Vdc}}<1$. Hence, for HAPF using conventional 3-phase VSI as well as using nine switch inverter, capacitor dc-link voltage, $\mathrm{V}_{\mathrm{dc}}<2$ $\sqrt{2} \mathrm{Vx}$.

\section{CONCLUSION}

As technology advances, the demand for exponential electricity increases. Many consumer devices constantly demand quality performance for their operation. The performance of the devices depends to a large extent on the quality of the power supply. However, the quality of the end user's energy is influenced by various external and internal factors. They are like fluctuations of voltage and frequency, faults, faults, etc. These energy quality problems reduce the life and efficiency of the equipment. Therefore, these problems should be mitigated to improve the performance of the consumer equipment and the overall performance of the system.

Improving the quality of energy can be achieved by using a more efficient power filter and algorithm in a networked system. The work can be extended to improve the quality of the network fed to the network through renewable energy sources.

\section{REFERENCES}

[1] Soumya Ranjan Das, P.K. Ray, Asit Mohanty "Improvement in Power Quality using Hybrid Power Filters based on RLS Algorithm ",2017 International Conference on Alternative Energy in Developing Countries and Emerging Economies, 2017 AEDCEE, 25-26 May 2017, Bangkok, Thailand.

[2] S.Haugan and E.Tedeschi, "Reactive and Harmonic Compensation Using the Conservative Power Theory," International Conference on Ecological Vehicles and Renewable Energies (EVER). 2015

[3] O.P.Mahela and A.G.Shaik, "Topological aspects of power quality improvement techniques: A comprehensive overview," Renewable and Sustainable EnergyReviews,Elsevier. Vol. 58, 2016.

[4] Y.Jiang, J.Chang and S.Tian, "Multi-objective Optimal Design of Hybrid Active Power Filter," International Conference on Advanced Manufacture Technology and Industrial Application. 2016.

[5] L.Yilmaz, E.Durna and and M. Ermis, "Design and Implementation of a Hybrid System for the Mitigation of PQ Problems of MediumFrequency Induction Steel-Melting Furnaces," IEEE Transactions on Industry Applications Vol.52.2016.

[6] Q.N.Trinh and H.H Lee, "Versatile Shunt Hybrid Power Filter to Simultaneously Compensate Harmonic Currents and Reactive Power," Journals of Electrical Engineering and Technol.Vol.10(3),2015.

[7] P.Thirumoorthi and N.Yadaiah, "Design of current source hybrid power filter for harmonic current compensation," Simulation Modelling Practice and Theory, Elsevier.Vol.52.2015.

[8] V. Rashmi, M. Khare, "Study of Cascaded H-Bridge Converter Control Strategies and their Impact on Switching Harmonics", IJOSCIENCE, Vol. 4, Issue 3, 2018. Available at : http://ijoscience.com/ojsscience/index.php/ojsscience/article/vie $\mathrm{w} / 128 / 105$.

[9] D. Li, K. Yang, Z.Q. Zhu and Y. Qin "“A Novel Series Power Quality Controller with Reduced Passive Power Filter," IEEE Transactions On Industrial Electronics.January 14, 2016.

[10] L. R. Limongi, L. R. da Silva Filho, L. G. Genu, F. Bradaschia, and M. C. Cavalcanti, "Transformerless hybrid power filter based on a six-switch two-leg inverter for improved harmonic compensation performance," IEEE Transactions on Industrial Electronics, vol. 62, no. 1, pp. 40-51, 2015.

[11] E. Sundaram and M. Venugopal, "Design and implementation of three phase three level shunt active power filter for harmonic reduction," in Industrial Electronics Society, IECON 2014-40th Annual Conference of the IEEE. IEEE, 2014, pp. 1377-1383. 